\title{
Recent Advances in the Neurochemistry of Epilepsy
}

\author{
a report by
}

\section{Germán Sierra-Paredes}

Associate Professor, Department of Biochemistry and Molecular Biology, School of Medicine, University of Santiago

Epilepsy is a common neurological disorder, affecting $1-2 \%$ of the population worldwide, and is characterised by recurrent spontaneous seizures. Seizures are brief behavioural changes caused by the abnormally synchronous and rhythmic firing of neuronal assemblies in the brain, which may either involve specific systems of the brain or start in a restricted area and spread to involve multiple cortical and subcortical circuits. Epilepsies may result from long-lasting plastic changes in the brain affecting neurotransmitter release and transport, the properties of receptors and channels, regulation of gene expression, synaptic re-organisation and astrocyte activity. There is considerable evidence of ion channel alterations being the origin of the paroxysmal depolarisation shifts that initiate epileptic activity; however, recent studies on synaptic and non-synaptic transmission, the ion channels interactome, intracellular signalling pathways and glia-neuron signalling suggest that many neurochemical pathways play an important role in seizure initiation, maintenance and arrest. This article addresses some recent advances in our understanding of the neurochemical basis of the seizures that are suggesting new targets for antiepileptic therapy.

\section{Ion Channels Interactome}

Changes in ionic currents that flow through sodium, potassium, chloride and calcium channels have been proposed to be involved in epileptogenesis. ${ }^{1,2}$ The blockade of $\gamma$-aminobutyric acid (GABA) and glycine-mediated inhibitory neurotransmission or the potentiation of excitatory neurotransmission can induce generalised phasic activity resembling paroxysmal depolarisation shifts. Similar effects are seen with epileptogenic agents that act on the intrinsic mechanisms underlying membrane excitability, such as increasing sodium or calcium depolarising currents or reducing hyperpolarising potassium currents. In addition, the majority of current antiepileptic drugs are thought to modify excitatory and inhibitory neurotransmission through effects on voltage-gated ion channels, $\mathrm{GABA}_{\mathrm{A}}$ receptors and glutamate-mediated excitatory neurotransmission. ${ }^{3,4}$

However, as structure-function analyses in both physiological and pathophysiological conditions have shown potentially epileptogenic changes in the structure of ion channels in only a minority of human

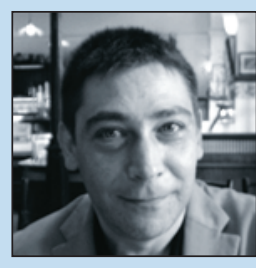

Germán Sierra-Paredes is an Associate Professor in the Department of Biochemistry and Molecular Biology of the School of Medicine at the University of Santiago de Compostela, and Principal Investigator of the Neurochemistry of Epilepsy Research Laboratory at the University of Santiago de Compostela.

E: bngersp@usc.es epilepsies, ${ }^{1}$ much recent research has focused on a variety of intracellular proteins that somehow interact with ion channels and receptors.

\section{Anchoring Proteins}

Many proteins are involved in the anchoring and trafficking of ion channels. A large group of these proteins are found in post-synaptic densities, including spectrin, actin, calpain, calcineurin, contactin, PSD-95, SAP-90, gephyrin, acidic calponin, Homer and several protein kinases. The expression of many of these molecules changes in epileptic tissue and in diverse experimental models of epilepsy. ${ }^{3}$

Studies performed on in vitro preparations and in cell cultures showed that glutamate receptors are anchored in the dendritic spines by an actin cytoskeleton. The mechanism for receptor redistribution involves calcium-mediated actin depolymerisation, and is related to rapid dendritic spine plasticity. This receptor redistribution has been shown to participate in pathological processes leading to epileptogenesis. ${ }^{5}$ When perfused in conscious, freely moving animals, the actin-disrupting agents latrunculin A and jasplakinolide modify neuronal excitability and lead to epileptic seizures. ${ }^{6}$

The permanent decrease in the seizure threshold observed after latrunculin A microperfusion ${ }^{6}$ is more likely to be related to morphological changes in the number or shape of dendritic spines, or a permanent re-organisation in the location of glutamate receptors or other proteins within the post-synaptic density that are highly dependent on F-actin for their localisation, such as CaMKII, spectrin, myosin $V, \alpha$-adducin, neurabin, neurabinll/spinophilin, cortactin and many others. The major functions of those actin-associated component proteins of the post-synaptic membrane appear to be signal transduction and modification of the microfilament arrays in response to synaptic activation, events thought to mediate long-term synaptic plasticity.

Synaptic glutamate receptors are concentrated in the post-synaptic complex of central synapses. This implies a highly organised and stable post-synaptic membrane with tightly anchored receptors. The identification of many anchoring proteins, such as rapsyn, gephyrin and PSD-95, and the association of post-synaptic density proteins into a molecular scaffold havestrengthened this view. Nevertheless, recent investigations suggest that the alpha-amino-3-hydroxy-5-methyl-4-isoxazolepropionic acid (AMPA) and n-methyl-D-aspartic acid (NMDA) receptors at synapses are highly dynamic. It is becoming evident that scaffolding proteins have many more dynamic properties than simply acting as inert scaffolds for other molecules. ${ }^{7}$ Both the rapid incorporation of AMPA and NMDA receptors into synapses and their removal by endocytosis are activity-dependent, suggesting that this exchange may underlie several forms of synaptic 
plasticity. Tovar and Westbrook ${ }^{8}$ demonstrated that NMDA receptors move laterally between synaptic and extrasynaptic pools, providing an alternative mechanism for altering the synaptic strength over the course of minutes.

These data suggest a sequence of events that may be involved in the longterm response of a post-synaptic neuron, such as increased excitatory activity in the pre-synaptic neuron, calcium influx, phosphatases and/or protein kinase activation, phosphorylation/dephosphorylation of receptors, ion channels and other proteins, cytoskeletal changes, mobilisation of receptor and ion channels and, finally, long-term changes in post-synaptic excitability. If this reflects the physiological sequence leading to biochemical and morphological changes involved in the normal function of neuronal networks, it is possible that biochemical and/or morphological alterations in any of these steps may produce pathological consequences. For instance, deregulated mobility caused by alterations in receptor subunit composition, protein kinases/phosphatases or cytoskeletal proteins is likely to be involved in hyperexcitability leading to epileptic seizures. ${ }^{9}$

\section{Calcineurin}

Calcineurin (CaN), also known as protein phosphatase $2 \mathrm{~B}$, is a calcium/ calmodulin-dependent phosphatase highly enriched in neural tissue. CaNmediated dephosphorylation is an important modulatory factor in many cellular processes, including development of learning and memory, regulation of neuronal plasticity and induction of apoptosis. CaN enzymatic activity has been related to epileptic seizures in several animal models. The increased intracellular calcium associated with status epilepticus could be responsible for activating $\mathrm{CaN}$ above its normal physiological level, because status epilepticus induces a loss of function of the endoplasmic reticulum $\mathrm{Mg}^{2+} / \mathrm{Ca}^{2+}$ ATPase. ${ }^{10}$ This enzyme sequesters calcium ions into the microsomes of the smooth endoplasmic reticulum, providing a high-affinity mechanism for regulating intracellular calcium concentration. After status epilepticus, ATPase-mediated uptake of calcium into the microsomes is less efficient, ${ }^{10}$ which could potentially result in higher than normal resting calcium concentrations inside the cell, affecting the status epilepticusinduced increase in CaN dephosphorylation.

One important $\mathrm{CaN}$-mediated mechanism is modulation of the $\mathrm{GABA}_{A}$ receptor. $G A B A_{A}$ receptors are the primary receptors responsible for the fast inhibitory response in neuronal tissue and play a major role in preventing the neuronal hyperexcitability associated with epilepsy. Furthermore, CaN may play a role in regulating the long-term changes that lead to epileptogenesis.

\section{Extrasynaptic communication appears}

to be as complex and dynamic as synaptic transmission, and a variety of extrasynaptic receptors for amino acids have been identified.

CaN inhibitors may acutely potentiate GABAergic transmission - thus transiently increasing the picrotoxin seizure threshold ${ }^{11}$ - but when excessive excitatory activity induces massive $\mathrm{Ca}^{2+}$ entry through NMDA or $\mathrm{Ca}^{2+}$ permeable AMPA receptors, CaN may be also involved in the activation of long-term molecular cellular mechanisms that lead to the sustained recurrent excitatory activity, which induces late spontaneous seizures.
CaN activity was significantly increased in the short and long term after actin depolymerisation with latrunculin A. The increase in CaN activity was reversed by continuous MK-801 microperfusion in the rat hippocampus in both acute and chronic animals. ${ }^{12}$ The increase in CaN activity observed after the in vivo microperfusion of latrunculin $\mathrm{A}$ in the rat hippocampus may be related to cytoskeletal re-organisation induced by F-actin depolymerisation followed by an increase in NMDA-receptor activation, with implications for epileptogenesis.

\section{Non-synaptic Transmission in Epilepsy}

The distinction between synaptic and non-synaptic transmission is a general property of amino acid and other neurotransmitter systems in the
Neurons can rapidly modulate synaptic $\mathrm{GABA}_{\mathrm{A}}$ receptor numbers at inhibitory synapses by controlling the availability and stability of extrasynaptic receptors.

mammalian brain. In recent years it has been increasingly evident that glutamate, GABA and glycine receptors, in addition to their subsynaptic localisation, are also expressed extrasynaptically. ${ }^{13}$

Extrasynaptic communication appears to be as complex and dynamic as synaptic transmission, and a variety of extrasynaptic receptors for amino acids have been identified. Metabotropic glutamate receptors are mainly located extrasynaptically. Non-synaptically localised AMPA receptors have been found in CA1 pyramidal neurons, in the dendritic layers of the hippocampus and in the molecular layer of the dentate gyrus, where pyramidal cell somata, dendrites and spines contain a large number of extrasynaptic receptors. The NMDA receptor subunit NR2B predominates at non-synaptic sites in hippocampal neurons. In cerebellar Golgi cells, the NMDA receptor subunit NR2D seems to be restricted to non-synaptic sites. Interestingly, incorporation of this subunit into various subunit composition NMDA receptors may cause loss of targeting to the post-synaptic membrane, suggesting that the actual balance between synaptic/nonsynaptic NMDA receptor functions can be regulated by specific subunit expression. ${ }^{14}$ Extrasynaptic $G_{A B A}$ and $G A B A_{B}$ receptors have been also unequivocally recognised. The single-channel properties of extrasynaptic $G_{A B A_{A}}$ receptors have been shown to be different from those within the synapse, and the $\delta$-subunit-containing $G_{A B A}$ receptors that mediate tonic inhibition are found only in extrasynaptic membranes..$^{15}$ All of these extrasynaptic receptors appear to be there for the specific purpose of sampling the low levels of amino acids present in the intercellular space. Some of them are probably activated during periods of intense stimulation, and may play a role in neuronal synchronisation.

In addition, recent research on receptor mobility shows that glutamate and GABA receptors are able to move between synaptic and extrasynaptic locations. The number and composition of glutamate and GABA receptors in or outside the synapse can be modulated by several factors. For instance, the localisation of NMDA receptors at synaptic sites is achieved through interactions between their intracellular domains, cytoskeletal elements and other cytoplasmically located 
submembrane proteins in the post-synaptic density, many of them scaffolding proteins. F-actin may be responsible for targeting NMDA receptors to synaptic sites because treatment with actin-depolymerising agents selectively reduces the numbers of synaptic NMDA-receptor clusters without affecting non-synaptic clusters.

In addition to lateral movements, GABA and glutamate receptors also undergo significant rates of constitutive endocytosis and recycling, which can directly modulate the efficacy of synaptic transmission. For $G_{A B A}$ receptors, synaptic confinement is at least partially facilitated by the postsynaptic scaffold protein gephyrin, which has recently been shown to

\section{It seems clear now that}

neurotransmitter receptors and

synapses are not fixed structures, but

rather are subjected to continuous

changes and replacements. photolysis of caged $\mathrm{Ca}^{2+}$ in astrocytes, ${ }^{18}$ suggesting that prolonged episodes of neuronal depolarisation evoked by astrocytic glutamate release contribute to epileptiform discharges. Recent data on gene expression in human temporal lobe epilepsy are consistent with an increased glutamate release from astrocytes. ${ }^{19}$

Synaptic and non-synaptic signals should be precisely integrated by the neurons. It is probable that changes in synaptic activity may modify the number, distribution and subunit composition of extrasynaptic amino acid receptors, and vice versa. Alterations in cross-talking among synaptic and non-synaptic transmission may be involved in several pathologies, including epilepsy.

\section{Intracellular Signalling Pathways and \\ Regulation of Gene Expression}

Recent evidence shows that activation of extracellular regulated kinase (ERK) and $p 38 \alpha$ mitogen-activated protein kinase (p38) pathways play a critical role in some forms of human epilepsy. The ERK kinases are abundantly expressed in the central nervous system, and are activated in response to various physiological stimuli associated with synaptic activity and plasticity, most notably calcium influx and neurotrophins, but also during pathological events such as brain ischaemia and epilepsy. ${ }^{20-22}$ ERK and p38 are involved in hippocampal seizure tolerance, ${ }^{23}$ and ERK activation appears to be sufficient to trigger epilepsy in some animal models.22,23 Pre-treatment with the ERK inhibitor PD98059 and the p38 inhibitor SB203580 selectively reduces seizure elicited activation of ERK and p38, respectively, and significantly reduces priming seizure-induced protection of CA3 neurons. ${ }^{20}$

\section{Conclusions}

The biochemical study of the ion channels interactome such as actindependent receptor-channel anchoring, related transduction pathways and many other post-synaptic density (PSD) proteins seems to be an important approach to the neuropathology and neuropharmacology of epilepsy. It seems clear now that neurotransmitter receptors and synapses are not fixed structures, but rather are subjected to continuous changes and replacements. Receptor and enzyme location seems to be a decisive factor for the transmission of neural messages among neurons, and it is probable that changes in receptor location may be involved in learning associated plasticity changes as well as in some brain diseases, including the epilepsies.
1. Avanzini A, Franceschetti S, Cellular biology of epileptogenesis, Lancet Neurology, 2003;2:33-42.

2. Avanzini A, Franceschetti S, Mantegazza M, Epileptogenic channelopathies: Experimental models of human pathologies, Epilepsia, 2007;48(Suppl. 2):51-64.

3. Meldrum BS, Rogawski MA, Molecular targets for antiepileptic drug development, Neurotherapeutics, 2007;4;18-61.

4. White HS, Smith MD, Wilcox KS, Mechanisms of Action of Antiepileptic Drugs, Int Rev Neurobiol, 2007;81:85-110.

5. Sierra-Paredes G, Sierra-Marcuño G, Extrasynaptic GABA and glutamate receptors in epilepsy, Curr Drug Targets CNS Neurol Disord, 2007;6:288-300.

6. Sierra-Paredes G, Oreiro-García MT, Núñez-Rodríguez A, et al., Seizures induced by in vivo latrunculin $A$ and jasplakinolide microperfusion in the rat hippocampus, J Mol Neurosci, 2006; 28:151-60.

7. Smythies J, The Dynamic Neuron, Cambridge, Massachusetts: The MIT Press, 2002

8. Tovar KR, Westbrook GL, The incorporation of NMDA receptors with a distinct subunit composition at nascent hippocampal synapses in vitro, J Neurosci, 1999;19:4180-88.

9. Sierra-Paredes G, Vázquez-López A, Oreiro-García MT, et al., Neurochemistry of epileptic seizures: the role of f-actin, intercellular glutamate and glutamate ionotropic receptors location. In: KJ Hollaway (ed.), New Research on Epilepsy and Behavior, New York: Nova Biomedical, 2007;57-93.

10. Parsons JT, Churn SB, Kochan LD, De-Lorenzo RJ, Pilocarpineinduced status epilepticus causes $\mathrm{N}$-metyl-D-aspartate receptor-dependent inhibition of microsomal $\mathrm{Mg}^{2+} / \mathrm{Ca}^{2}$ ATPase mediated $\mathrm{Ca}^{2+}$ uptake, J Neurochem, 2000;74: 1209-18.

11. Vázquez-López A, Sierra-Paredes G, Sierra-Marcuño G, Anticonvulsant effect of the calcineurin inhibitor ascomycin on seizures induced by picrotoxin microperfusion in the rat hippocampus, Pharmacol Biochem Behav, 2006;84:511-16.

12. Sierra-Paredes G, Sierra-Marcuño G, Ascomycin and FK 506: Pharmacology and therapeutic potential as anticonvulsants and neuroprotectants, CNS: Neuroscience \& Therapeutics, 2008;14: $36-46$.

13. Vizi ES, Mike A, Nonsynaptic receptors for GABA and glutamate, Curr Top Med Chem, 2006;6:941-8.

14. Vizi ES, Kiss JP, Lendvai B, Nonsynaptic communication in the central nervous system, Neurochem Int, 2004;45:443-51.

15. Nusser Z, Mody I, Selective modulation of tonic and phasic inhibitions in dentate gyrus granule cells, J Neurophysiol, 2002; 87:2624-8.
16. Knuesel I, Zuellig RA, Schaub MC, Fritschy JM, Alterations in dystrophin and utrophin expression parallel the reorganization of GABAergic synapses in a mouse model of temporal lobe epilepsy, Eur J Neurosci, 2001;13:1113-24.

17. Wetherington J, Serrano G, Dingledine R, Astrocytes in the epileptic brain, Neuron, 2008;58:168-78.

18. Tian GF, Azmi H, Takano T, et al., An astrocytic basis of epilepsy, Nat Med, 2005;11;973-81.

19. Lee TS, Mane S, Eid T, et al., Gene expression in temporal lobe epilepsy is consistent with increased release of glutamate by astrocytes, Mol Med, 2007;13:1-13.

20. Nateri AS, Raivich G, Gebhardt C, et al., ERK activation causes epilepsy by stimulating NMDA receptor activity, EMBO J, 2007; 26:4891-4901.

21. Namiki K, Nakamura A, Furuya M, et al., Involvement of p38alplha in Kainate-Induced Seizure and Neuronal Cell Damage, I Recept Signal Transduct Res, 2007;27:99-111.

22. Merlo D, Cifelli P, Cicconi S, et al., 4-Aminopyridine-induced epileptogenesis depends on activation of mitogen-activated protein kinase ERK, J Neurochem, 2004;89:654-9.

23. Jiang W, Van Cleemput J, Sheerin AH, et al., Involvement of Extracellular Regulated Kinase and p38 Kinase in Hippocampal Seizure Tolerance, J Neurosci Res, 2005;81:581-8. 